\title{
On Direct Integral Expansion for Periodic Block-Operator Jacobi Matrices and Applications
}

Leonid GOLINSKII ${ }^{\dagger}$ and Anton KUTSENKO ${ }^{\ddagger}$

$\dagger^{\dagger}$ B. Verkin Institute for Low Temperature Physics and Engineering, 47 Science Ave., Kharkiv 61103, Ukraine

E-mail: golinskii@ilt.kharkov.ua

$\ddagger$ Jacobs University, Campus Ring 1, 28759 Bremen, Germany

E-mail: akucenko@gmail.com

Received December 03, 2018, in final form June 23, 2019; Published online July 02, 2019 https://doi.org/10.3842/SIGMA.2019.050

\begin{abstract}
We construct a functional model (direct integral expansion) and study the spectra of certain periodic block-operator Jacobi matrices, in particular, of general 2D partial difference operators of the second order. We obtain the upper bound, optimal in a sense, for the Lebesgue measure of their spectra. The examples of the operators for which there are several gaps in the spectrum are given.
\end{abstract}

Key words: functional model; block Jacobi matrices; partial difference operators; periodicity; spectrum

2010 Mathematics Subject Classification: 47B36; 47B39; 35P15

\section{Introduction}

Consider a block-operator Jacobi matrix on the Hilbert space $\mathcal{F}=\ell^{2}(\mathbb{Z}, \mathcal{H})$

$$
(J u)_{q}:=A_{q-1} u_{q-1}+B_{q} u_{q}+A_{q}^{*} u_{q+1}, \quad B_{q}=B_{q}^{*}, \quad q \in \mathbb{Z}, \quad u_{q} \in \mathcal{H},
$$

where the blocks $\left\{A_{q}, B_{q}\right\}$ are bounded linear operators on the Hilbert space $\mathcal{H}$. Under the standard assumption $\sup _{q \in \mathbb{Z}}\left(\left\|A_{q}\right\|+\left\|B_{q}\right\|\right)<\infty$ on the entries, the matrix $J$ generates a bounded and self-adjoint operator $J$ on $\mathcal{F}$.

We are primarily interested in the case when $\mathcal{H}=\ell^{2}(\mathbb{Z})$, and the blocks $B_{q}, A_{q}$ are themselves Hermitian (not necessarily real symmetric) 1D Jacobi matrices

$$
(J u)_{q}:=A_{q-1} u_{q-1}+B_{q} u_{q}+A_{q} u_{q+1}, \quad q \in \mathbb{Z}, \quad u_{q}=\left(u_{q}(l)\right)_{l} \in \ell^{2}(\mathbb{Z}) .
$$

The block matrices of this type are known as Jacobi-block-Jacobi matrices. So,

$$
A_{q}=J\left(\left\{a_{q, r}\right\}_{r \in \mathbb{Z}},\left\{\alpha_{q, r}\right\}_{r \in \mathbb{Z}}\right), \quad B_{q}=J\left(\left\{c_{q, r}\right\}_{r \in \mathbb{Z}},\left\{b_{q, r}\right\}_{r \in \mathbb{Z}}\right),
$$

with real entries $\left\{a_{q, r}\right\}\left(\left\{c_{q, r}\right\}\right)$ along the main diagonal, and complex entries $\left\{\alpha_{q, r}\right\}\left(\left\{b_{q, r}\right\}\right)$ and their conjugates along the diagonals above and below the main one, respectively.

Such block-operator model arises when we deal with 2D partial difference operators $L$ of the second order of the form

$$
(L \widetilde{u})_{i k}:=a_{i-1, k} u_{i-1, k}+a_{i, k} u_{i+1, k}+b_{i, k-1} u_{i, k-1}+b_{i, k} u_{i, k+1}+c_{i, k} u_{i, k},
$$

on the Hilbert space $\ell^{2}\left(\mathbb{Z}^{2}\right)$. A natural isometry $U_{0}$ between $\ell^{2}\left(\mathbb{Z}^{2}\right)$ and $\mathcal{F}$

$$
\widetilde{u}=\left(u_{i, k}\right)_{i, k \in \mathbb{Z}} \rightarrow u=U_{0} \widetilde{u}=\left(u_{j}\right)_{j \in \mathbb{Z}}: u_{j}=\left(\ldots, u_{j,-1}, u_{j, 0}, u_{j, 1}, \ldots\right)^{\prime}
$$


transforms the operator $L$ in (1.4) into $J:=U_{0} L U_{0}^{-1}$ on $\mathcal{F}$, where

$$
\begin{aligned}
& (J u)_{j}=A_{j-1} u_{j-1}+B_{j} u_{j}+A_{j} u_{j+1}, \quad j \in \mathbb{Z}, \\
& A_{j}:=\operatorname{diag}\left(a_{j, k}\right)_{k \in \mathbb{Z}}, \quad B_{j}:=J\left(\left\{c_{j, k}\right\}_{k \in \mathbb{Z}},\left\{b_{j, k}\right\}_{k \in \mathbb{Z}}\right),
\end{aligned}
$$

see, e.g., [1, Section VII.3]. This is a special case of the model operator $J(1.2)$ with $\alpha_{j, k}=0$. In particular, for $2 \mathrm{D}$ discrete Schrödinger operators $H\left(a_{j, k}=b_{j, k}=1\right)$ we have

$$
(H \widetilde{u})_{i, k}:=u_{i-1, k}+u_{i+1, k}+u_{i, k-1}+u_{i, k+1}+c_{i, k} u_{i, k},
$$

SO

$$
A_{j}=I, \quad B_{j}=J\left(\left\{c_{j, k}\right\}_{k \in \mathbb{Z}},\{1\}\right),
$$

1D discrete Schrödinger operators.

Let $p_{j} \in \mathbb{N}, j=1,2$. A sequence of complex numbers $\left\{w_{q, r}\right\}_{q, r \in \mathbb{Z}}$ is called $\left(p_{1}, p_{2}\right)$-periodic if

$$
w_{q+k_{1} p_{1}, r+k_{2} p_{2}}=w_{q, r}, \quad \forall q, r, k_{1}, k_{2} \in \mathbb{Z} .
$$

The operator $J$ in (1.2) is called $\left(p_{1}, p_{2}\right)$-periodic if $\left\{a_{q, r}\right\},\left\{\alpha_{q, r}\right\},\left\{c_{q, r}\right\}$, and $\left\{b_{q, r}\right\}, q, r \in \mathbb{Z}$, are $\left(p_{1}, p_{2}\right)$-periodic. Equivalently,

1) all the blocks $A_{q}, B_{q}$ are $p_{2}$-periodic $1 \mathrm{D}$ Jacobi matrices;

2) $J$ is block periodic with period $p_{1}: A_{q+p_{1}}=A_{q}, B_{q+p_{1}}=B_{q}$.

We say that the partial difference operator $L(1.4)$ is $\left(p_{1}, p_{2}\right)$-periodic, if all the coefficients are $\left(p_{1}, p_{2}\right)$-periodic, or equivalently, $J(1.5)$ is $\left(p_{1}, p_{2}\right)$-periodic.

In Section 2 we recall a direct integral expansion (a functional model) for the $\left(p_{1}, p_{2}\right)$-periodic operator $J$ in (1.2)-(1.3) and establish the banded structure of its spectrum $\sigma(J)$. In Section 3 we estimate the Lebesgue measure of $\sigma(J)$.

Theorem 1.1. Let $p_{1}, p_{2} \geq 3$. The Lebesgue measure of the spectrum for the periodic operator $J$ in (1.2)-(1.3) admits the upper bound

$$
|\sigma(J)| \leq \min _{(m, n) \in \mathbb{Z}^{2}} R_{m, n}
$$

where

$$
R_{m, n}:=4 \sum_{j=1}^{p_{1}}\left(\left|b_{j, n}\right|+2\left|\alpha_{j, n}\right|\right)+4 \sum_{k=1}^{p_{2}}\left(\left|a_{m, k}\right|+2\left|\alpha_{m, k}\right|\right)-8\left|\alpha_{m, n}\right| .
$$

The sequence $\left\{R_{m, n}\right\}$ is $\left(p_{1}, p_{2}\right)$-periodic, so minimum in (1.7) is actually taken over the finite set of indices $m=1, \ldots, p_{1}$ and $n=1, \ldots, p_{2}$.

The similar result holds for $\min \left(p_{1}, p_{2}\right)=2$, see Remark 3.1 below.

Note that there is a simple general bound for $|\sigma(J)|$ (which has nothing to do with periodicity) based on the fact that $J$ is a three-diagonal block-matrix

$$
\begin{aligned}
|\sigma(J)| & \leq 2\|J\| \leq 2\left(2 \max _{n}\left\|A_{n}\right\|+\max _{n}\left\|B_{n}\right\|\right) \\
& \leq \max _{n}\left(8 \max _{m}\left|\alpha_{n, m}\right|+4 \max _{m}\left|a_{n, m}\right|\right)+\max _{n}\left(4 \max _{m}\left|b_{n, m}\right|+2 \max _{m}\left|c_{n, m}\right|\right) .
\end{aligned}
$$

The point is that certain parameters, such as $c_{n, m}$, which appear in (1.9), do not enter (1.7). So, once some values of $c_{n, m}$ are large enough, bound (1.7) is better that (1.9). On the other hand, (1.7) contains sums of the entries compared to (1.9), which does not. 
There is another upper bound for the length of the spectrum, based on Gershgorin's theorem, which is discussed in Remark 3.3.

For the 1D scalar, $p$-periodic Jacobi operator $J$ the estimates for the spectrum

$$
|\sigma(J)| \leq 4\left(a_{1} a_{2} \cdots a_{p}\right)^{1 / p}
$$

were obtained in $[7,9]$. Here $a_{n}$ are the off-diagonal entries of $J$. Recently the second author [11] improved this result to

$$
|\sigma(J)| \leq 4 \min _{n} a_{n}
$$

We see that both bounds do not depend on the diagonal entries, so the lack of $c_{m, n}$ in (1.7) looks reasonable.

Corollary 1.2. For a $2 D$ periodic, partial difference operators of the second order $L$ in (1.4) the spectral estimate is

$$
\frac{1}{4}|\sigma(L)| \leq \min _{n} \sum_{j=1}^{p_{1}}\left|b_{j, n}\right|+\min _{m} \sum_{k=1}^{p_{2}}\left|a_{m, k}\right| .
$$

In particular, $|\sigma(H)| \leq 4\left(p_{1}+p_{2}\right)$ for $2 D$ periodic, discrete Schrödinger operators $H$ (1.6).

The estimate $|\sigma(H)| \leq 4\left(p_{1}+p_{2}\right)$ was previously obtained in [10].

We complete the paper with examples of $2 \mathrm{D}$ discrete Schrödinger operators with explicitly computed spectra, and an operator, which has a maximal number of gaps in its spectrum.

\section{Direct integral expansion}

We begin with auxiliary, Hermitian matrix-functions of the order $p_{2}$

$$
\mathcal{A}_{n}\left(x_{2}\right)=\left[\begin{array}{cccccc}
a_{n, 1} & \alpha_{n, 1} & & & & \mathrm{e}^{\mathrm{i} x_{2}} \bar{\alpha}_{n, p_{2}} \\
\bar{\alpha}_{n, 1} & a_{n, 2} & \alpha_{n, 2} & & & \\
& \bar{\alpha}_{n, 2} & a_{n, 3} & \alpha_{n, 3} & & \\
& & \ddots & \ddots & \ddots & \\
& & & \bar{\alpha}_{n, p_{2}-2} & a_{n, p_{2}-1} & \alpha_{n, p_{2}-1} \\
\mathrm{e}^{-\mathrm{i} x_{2}} \alpha_{n, p_{2}} & & & & \bar{\alpha}_{n, p_{2}-1} & a_{n, p_{2}}
\end{array}\right]
$$

and

$$
\mathcal{B}_{n}\left(x_{2}\right)=\left[\begin{array}{cccccc}
c_{n, 1} & b_{n, 1} & & & & \mathrm{e}^{\mathrm{i} x_{2}} \bar{b}_{n, p_{2}} \\
\bar{b}_{n, 1} & c_{n, 2} & b_{n, 2} & & & \\
& \bar{b}_{n, 2} & c_{n, 3} & b_{n, 3} & & \\
& & \ddots & \ddots & \ddots & \\
& & & \bar{b}_{n, p_{2}-2} & c_{n, p_{2}-1} & b_{n, p_{2}-1} \\
\mathrm{e}^{-\mathrm{i} x_{2}} b_{n, p_{2}} & & & & \bar{b}_{n, p_{2}-1} & c_{n, p_{2}}
\end{array}\right],
$$

$x_{2} \in[0,2 \pi)$, and combine them in a single block matrix-function $\mathcal{S}$ of the order $p:=p_{1} p_{2}$,

$$
\mathcal{S}=\mathcal{S}\left(x_{1}, x_{2}\right)=\left[\begin{array}{cccccc}
\mathcal{B}_{1} & \mathcal{A}_{1} & & & & \mathrm{e}^{\mathrm{i} x_{1}} \mathcal{A}_{p_{1}} \\
\mathcal{A}_{1} & \mathcal{B}_{2} & \mathcal{A}_{2} & & & \\
& \mathcal{A}_{2} & \mathcal{B}_{3} & \mathcal{A}_{3} & & \\
& & \ddots & \ddots & \ddots & \\
& & & \mathcal{A}_{p_{1}-2} & \mathcal{B}_{p_{1}-1} & \mathcal{A}_{p_{1}-1} \\
\mathrm{e}^{-\mathrm{i} x_{1}} \mathcal{A}_{p_{1}} & & & & \mathcal{A}_{p_{1}-1} & \mathcal{B}_{p_{1}}
\end{array}\right],
$$

$x_{1}, x_{2} \in[0,2 \pi)$, the main object under consideration, known as a symbol. 
Denote $\mathbb{T}^{2}:=[0,2 \pi) \times[0,2 \pi)$, and put

$$
\mathcal{L}=\int_{\mathbb{T}^{2}}^{\oplus} \mathbb{C}^{p} \frac{\mathrm{d} x_{1} \mathrm{~d} x_{2}}{4 \pi^{2}}=L^{2}\left(\mathbb{T}^{2}, \mathbb{C}^{p}\right)=\left\{\left[\begin{array}{c}
g_{1}\left(x_{1}, x_{2}\right) \\
g_{2}\left(x_{1}, x_{2}\right) \\
\vdots \\
g_{p_{1}}\left(x_{1}, x_{2}\right)
\end{array}\right]: g_{k}\left(x_{1}, x_{2}\right)=\left[\begin{array}{c}
g_{k, 1}\left(x_{1}, x_{2}\right) \\
g_{k, 2}\left(x_{1}, x_{2}\right) \\
\vdots \\
g_{k, p_{2}}\left(x_{1}, x_{2}\right)
\end{array}\right]\right\},
$$

where

$$
\begin{aligned}
& g_{k, j}\left(x_{1}, x_{2}\right)=\sum_{m, n \in \mathbb{Z}} \hat{g}_{k, j}(m, n) \mathrm{e}^{\mathrm{i} m x_{1}+\mathrm{i} n x_{2}} \in L^{2}\left(\mathbb{T}^{2}\right), \\
& k=1,2, \ldots, p_{1}, \quad j=1,2, \ldots, p_{2} .
\end{aligned}
$$

We have

$$
\|g\|_{\mathcal{L}}^{2}=\sum_{k, j}\left\|g_{k, j}\right\|_{L^{2}\left(\mathbb{T}^{2}\right)}^{2}=\sum_{k, j, m, n}\left|\hat{g}_{k, j}(m, n)\right|^{2} .
$$

There is a natural isometry $U: \mathcal{L} \rightarrow \mathcal{F}=\ell^{2}\left(\mathbb{Z}, \ell^{2}(\mathbb{Z})\right)$ which acts by

$$
U\left[\begin{array}{c}
g_{1} \\
g_{2} \\
\vdots \\
g_{p_{1}}
\end{array}\right]=u=\left(u_{r}\right)_{r \in \mathbb{Z}}, \quad u_{r}=\left(u_{r}(s)\right)_{s \in \mathbb{Z}}: u_{k+p_{1} m}\left(j+p_{2} n\right)=\hat{g}_{k, j}(m, n) .
$$

Assume that $p_{1}, p_{2} \geq 3$. The above symbol $\mathcal{S}$ defines a multiplication operator $M(\mathcal{S})$ on $\mathcal{L}$ by

$$
M(\mathcal{S}) g=\mathcal{S}\left[\begin{array}{c}
g_{1}\left(x_{1}, x_{2}\right) \\
g_{2}\left(x_{1}, x_{2}\right) \\
\vdots \\
g_{p_{1}}\left(x_{1}, x_{2}\right)
\end{array}\right]=\left[\begin{array}{c}
h_{1}\left(x_{1}, x_{2}\right) \\
h_{2}\left(x_{1}, x_{2}\right) \\
\vdots \\
h_{p_{1}}\left(x_{1}, x_{2}\right)
\end{array}\right]
$$

with

$$
\begin{aligned}
& h_{1}\left(x_{1}, x_{2}\right)=\mathcal{B}_{1} g_{1}+\mathcal{A}_{1} g_{2}+\mathrm{e}^{\mathrm{i} x_{1}} \mathcal{A}_{p_{1}} g_{p_{1}}, \\
& h_{l}\left(x_{1}, x_{2}\right)=\mathcal{A}_{l-1} g_{l-1}+\mathcal{B}_{l} g_{l}+\mathcal{A}_{l} g_{l+1}, \quad l=2, \ldots, p_{1}-1, \\
& h_{p_{1}}\left(x_{1}, x_{2}\right)=\mathcal{A}_{p_{1}-1} g_{p_{1}-1}+\mathcal{B}_{p_{1}} g_{p_{1}}+\mathrm{e}^{-\mathrm{i} x_{1}} \mathcal{A}_{p_{1}} g_{1} .
\end{aligned}
$$

The result below is a cornerstone of $2 \mathrm{D}$ discrete, Floquet-Bloch theory, see [12, Section 5.3] for $1 \mathrm{D}$ theory (the calculation in dimension 2 is completely analogous).

Theorem 2.1. Let $p_{1}, p_{2} \geq 3$. The $\left(p_{1}, p_{2}\right)$-periodic operator $J(1.2)-(1.3)$ is unitarily equivalent to the multiplication operator $M(\mathcal{S})(2.5)-(2.6)$

$$
J=U M(\mathcal{S}) U^{-1},
$$

$U$ is defined in (2.4).

Remark 2.2. There is nothing special in the case $\min \left(p_{1}, p_{2}\right)=2$, but the symbol looks differently. Precisely, if $p_{1} \geq 3, p_{2}=2$, we have

$$
\begin{aligned}
& \mathcal{A}_{n}=\left[\begin{array}{cc}
a_{n, 1} & \alpha_{n, 1}+\mathrm{e}^{\mathrm{i} x_{2}} \bar{\alpha}_{n, 2} \\
\bar{\alpha}_{n, 1}+\mathrm{e}^{-\mathrm{i} x_{2}} \alpha_{n, 2} & a_{n, 2}
\end{array}\right], \\
& \mathcal{B}_{n}=\left[\begin{array}{cc}
c_{n, 1} & b_{n, 1}+\mathrm{e}^{\mathrm{i} x_{2}} \bar{b}_{n, 2} \\
\bar{b}_{n, 1}+\mathrm{e}^{-\mathrm{i} x_{2}} b_{n, 2} & c_{n, 2}
\end{array}\right], \quad n=1, \ldots, p_{1},
\end{aligned}
$$


and $\mathcal{S}$ is of the form (2.3). If $p_{1}=2, p_{2} \geq 3$, we take $\mathcal{A}_{1}, \mathcal{B}_{1}, \mathcal{A}_{2}, \mathcal{B}_{2}$ as in (2.1), (2.2), and

$$
\mathcal{S}\left(x_{1}, x_{2}\right)=\left[\begin{array}{cc}
\mathcal{B}_{1} & \mathcal{A}_{1}+\mathrm{e}^{\mathrm{i} x_{1}} \mathcal{A}_{2} \\
\mathcal{A}_{1}+\mathrm{e}^{-\mathrm{i} x_{1}} \mathcal{A}_{2} & \mathcal{B}_{2}
\end{array}\right] .
$$

Finally, if $p_{1}=p_{2}=2$, then $\mathcal{A}_{n}, \mathcal{B}_{n}$, and $\mathcal{S}$ are of the form (2.7)-(2.8).

Denote by $\lambda_{1} \geq \lambda_{2} \geq \cdots \geq \lambda_{p}, \lambda_{j}=\lambda_{j}\left(x_{1}, x_{2}\right)$, the set of all eigenvalues of $\mathcal{S}$, labeled in the non-increasing order. According to the general result on the spectrum of multiplication operators,

$$
\sigma(J)=\sigma(M(\mathcal{S}))=\bigcup_{k=1}^{p} \Lambda_{k}, \quad \Lambda_{k}:=\left[\inf _{\mathbb{T}^{2}} \lambda_{k}\left(x_{1}, x_{2}\right), \sup _{\mathbb{T}^{2}} \lambda_{k}\left(x_{1}, x_{2}\right)\right],
$$

the $k$ 's band in the spectrum. So we come to the following

Corollary 2.3. The spectrum of the $\left(p_{1}, p_{2}\right)$-periodic Jacobi-block-Jacobi matrix (1.2) has the banded structure

$$
\sigma(J)=\bigcup_{k=1}^{p} \Lambda_{k}, \quad p:=p_{1} p_{2},
$$

with the closed intervals $\Lambda_{k}$ (2.9). So, the number of the gaps in the spectrum does not exceed $p-1$.

Note that for a $\left(p_{1}, p_{2}\right)$-periodic, $2 \mathrm{D}$ discrete Schrödinger operator the symbol $\mathcal{S}$ takes the form

$$
\begin{aligned}
& \mathcal{S}\left(x_{1}, x_{2}\right)=\left[\begin{array}{cccccc}
\mathcal{B}_{1}\left(x_{2}\right) & I_{p_{2}} & & & & \mathrm{e}^{\mathrm{i} x_{1}} I_{p_{2}} \\
I_{p_{2}} & \mathcal{B}_{2}\left(x_{2}\right) & I_{p_{2}} & & & \\
& I_{p_{2}} & \mathcal{B}_{3}\left(x_{2}\right) & I_{p_{2}} & & \\
& & \ddots & \ddots & \ddots & \\
& & & I_{p_{2}} & \mathcal{B}_{p_{1}-1}\left(x_{2}\right) & I_{p_{2}} \\
\mathrm{e}^{-\mathrm{i} x_{1}} I_{p_{2}} & & & & I_{p_{2}} & \mathcal{B}_{p_{1}}\left(x_{2}\right)
\end{array}\right], \\
& \mathcal{B}_{n}\left(x_{2}\right)=\left[\begin{array}{cccccc}
c_{n, 1} & 1 & & & & \mathrm{e}^{\mathrm{i} x_{2}} \\
1 & c_{n, 2} & 1 & & & \\
& 1 & c_{n, 3} & 1 & & \\
& & \ddots & \ddots & \ddots & \\
& & & 1 & c_{n, p_{2}-1} & 1 \\
\mathrm{e}^{-\mathrm{i} x_{2}} & & & & 1 & c_{n, p_{2}}
\end{array}\right], \quad x_{1}, x_{2} \in[0,2 \pi) \text {. }
\end{aligned}
$$

\section{Spectral estimates for periodic block-Jacobi operators}

In this section we are aimed at proving Theorem 1.1. By Corollary 2.3,

$$
\sigma(J)=\sigma(M(\mathcal{S}))=\bigcup_{k=1}^{p} \Lambda_{k}, \quad \Lambda_{k}=\left[l_{k}, r_{k}\right]
$$

are the closed intervals, swept by the $k$-th eigenvalue $\lambda_{k}\left(x_{1}, x_{2}\right)$, arranged in the non-increasing order, as the pair $\left(x_{1}, x_{2}\right)$ runs over $\mathbb{T}^{2}$.

We are looking for two constant matrices $\mathcal{S}_{ \pm}$, i.e., independent of $\left(x_{1}, x_{2}\right)$, so that

$$
\mathcal{S}_{-} \leq \mathcal{S} \leq \mathcal{S}_{+} \quad \Rightarrow \quad \lambda_{k}^{-} \leq \lambda_{k}\left(x_{1}, x_{2}\right) \leq \lambda_{k}^{+},
$$


where $\lambda_{1}^{ \pm} \geq \lambda_{2}^{ \pm} \geq \cdots \geq \lambda_{p}^{ \pm}$are eigenvalues of $\mathcal{S}_{ \pm}$, arranged in the non-increasing order. Hence,

$$
|\sigma(M(\mathcal{S}))| \leq \sum_{k=1}^{p}\left(r_{k}-l_{k}\right) \leq \sum_{k=1}^{p}\left(\lambda_{k}^{+}-\lambda_{k}^{-}\right)=\operatorname{tr}\left(\mathcal{S}_{+}-\mathcal{S}_{-}\right)
$$

To this end put

$$
\begin{aligned}
& \mathcal{A}_{n}^{1}=\left[\begin{array}{cccccc}
a_{n, 1} & \alpha_{n, 1} & & & & \\
\bar{\alpha}_{n, 1} & a_{n, 2} & \alpha_{n, 2} & & & \\
& \bar{\alpha}_{n, 2} & a_{n, 3} & \alpha_{n, 3} & & \\
& & \ddots & \ddots & \ddots & \\
& & & \bar{\alpha}_{n, p_{2}-2} & a_{n, p_{2}-1} & \alpha_{n, p_{2}-1} \\
& & & & \bar{\alpha}_{n, p_{2}-1} & a_{n, p_{2}}
\end{array}\right], \\
& \mathcal{A}_{n}^{2}\left(x_{2}\right)=\left[\begin{array}{cc}
\mathrm{e}^{\mathrm{i} x_{2}} \bar{\alpha}_{n, p_{2}} \\
\mathrm{e}^{-\mathrm{i} x_{2}} \alpha_{n, p_{2}}
\end{array}\right],
\end{aligned}
$$

so $\mathcal{A}_{n}^{1}$ and $\mathcal{A}_{n}^{2}$ are Hermitian matrices, and let $\mathcal{A}_{n}=\mathcal{A}_{n}^{1}+\mathcal{A}_{n}^{2}$. Similarly,

$$
\begin{aligned}
& \mathcal{B}_{n}^{1}=\left[\begin{array}{cccccc}
c_{n, 1} & b_{n, 1} & & & & \\
\bar{b}_{n, 1} & c_{n, 2} & b_{n, 2} & & & \\
& \bar{b}_{n, 2} & c_{n, 3} & b_{n, 3} & & \\
& & \ddots & \ddots & \ddots & \\
& & & \bar{b}_{n, p_{2}-2} & c_{n, p_{2}-1} & b_{n, p_{2}-1} \\
& & & & \bar{b}_{n, p_{2}-1} & c_{n, p_{2}}
\end{array}\right], \\
& \mathcal{B}_{n}^{2}\left(x_{2}\right)=\left[\begin{array}{cc} 
& \mathrm{e}^{\mathrm{i} x_{2}} \bar{b}_{n, p_{2}} \\
\mathrm{e}^{-\mathrm{i} x_{2}} b_{n, p_{2}} & \\
\end{array}\right],
\end{aligned}
$$

so $\mathcal{B}_{n}^{1}$ and $\mathcal{B}_{n}^{2}$ are Hermitian matrices, and let $\mathcal{B}_{n}=\mathcal{B}_{n}^{1}+\mathcal{B}_{n}^{2}$. It follows that

$$
\mathcal{S}\left(x_{1}, x_{2}\right)=\mathcal{S}_{1}+\mathcal{S}_{2}\left(x_{1}, x_{2}\right)
$$

with

$$
\mathcal{S}_{1}=\left[\begin{array}{cccccc}
\mathcal{B}_{1}^{1} & \mathcal{A}_{1}^{1} & & & & \\
\mathcal{A}_{1}^{1} & \mathcal{B}_{2}^{1} & \mathcal{A}_{2}^{1} & & & \\
& \mathcal{A}_{2}^{1} & \mathcal{B}_{3}^{1} & \mathcal{A}_{3}^{1} & & \\
& & \ddots & \ddots & \ddots & \\
& & & \mathcal{A}_{p_{1}-2}^{1} & \mathcal{B}_{p_{1}-1}^{1} & \mathcal{A}_{p_{1}-1}^{1} \\
& & & & \mathcal{A}_{p_{1}-1}^{1} & \mathcal{B}_{p_{1}}^{1}
\end{array}\right]
$$




$$
\mathcal{S}_{2}\left(x_{1}, x_{2}\right)=\left[\begin{array}{cccccc}
\mathcal{B}_{1}^{2} & \mathcal{A}_{1}^{2} & & & & \mathrm{e}^{\mathrm{i} x_{1}} \mathcal{A}_{p_{1}} \\
\mathcal{A}_{1}^{2} & \mathcal{B}_{2}^{2} & \mathcal{A}_{2}^{2} & & & \\
& \mathcal{A}_{2}^{2} & \mathcal{B}_{3}^{2} & \mathcal{A}_{3}^{2} & & \\
& & \ddots & \ddots & \ddots & \\
& & & \mathcal{A}_{p_{1}-2}^{2} & \mathcal{B}_{p_{1}-1}^{2} & \mathcal{A}_{p_{1}-1}^{2} \\
\mathrm{e}^{-\mathrm{i} x_{1}} \mathcal{A}_{p_{1}} & & & & \mathcal{A}_{p_{1}-1}^{2} & \mathcal{B}_{p_{1}}^{2}
\end{array}\right] .
$$

In decomposition (3.4) of the symbol, $\mathcal{S}_{1}$ is a constant matrix, and $\mathcal{S}_{2}$ is sparse, i.e., it contains few nonzero entries.

Next,

$$
\mathcal{S}_{2}=\mathcal{S}_{3}+\mathcal{S}_{4}+\sum_{j=1}^{p_{1}-1} E_{j}
$$

with

$$
\mathcal{S}_{3}:=\operatorname{diag}\left(\mathcal{B}_{1}^{2}, \ldots, \mathcal{B}_{p_{1}}^{2}\right), \quad \mathcal{S}_{4}:=\left[\begin{array}{cc}
\mathrm{e}^{\mathrm{i} x_{1}} \mathcal{A}_{p_{1}} \\
\mathrm{e}^{-\mathrm{i} x_{1}} \mathcal{A}_{p_{1}}
\end{array}\right],
$$

and

$$
\begin{aligned}
& E_{1}:= {\left[\begin{array}{ccccc}
0 & \mathcal{A}_{1}^{2} & 0 & & \\
\mathcal{A}_{1}^{2} & 0 & 0 & \ddots & \\
0 & 0 & 0 & \ddots & \\
& \ddots & \ddots & \ddots & \ddots \\
& & & &
\end{array}\right], \quad E_{2}:=\left[\begin{array}{ccccc}
0 & 0 & 0 & & \\
0 & 0 & \mathcal{A}_{2}^{2} & \ddots & \\
0 & \mathcal{A}_{2}^{2} & 0 & \ddots & \\
& \ddots & \ddots & \ddots & \ddots \\
& & & &
\end{array}\right], } \\
& E_{p_{1}-1}:=\left[\begin{array}{cccc}
\ddots & \ddots & \ddots & \ddots \\
\ddots & 0 & 0 & 0 \\
\ddots & 0 & 0 & \mathcal{A}_{p_{1}-1}^{2} \\
\ddots & 0 & \mathcal{A}_{p_{1}-1}^{2} & 0
\end{array}\right] .
\end{aligned}
$$

Since $\mathcal{A}_{p_{1}}=G_{1}+G_{1}^{*}+G_{2}$ with

$$
G_{1}:=\left[\begin{array}{ccccc}
0 & & & & \mathrm{e}^{\mathrm{i} x_{2}} \bar{\alpha}_{p_{1}, p_{2}} \\
\bar{\alpha}_{p_{1}, 1} & 0 & & & \\
& \bar{\alpha}_{p_{1}, 2} & 0 & & \\
& & \ddots & \ddots & \\
& & & \bar{\alpha}_{p_{1}, p_{2}-1} & 0
\end{array}\right], \quad G_{2}:=\operatorname{diag}\left(a_{p_{1}, 1}, \ldots, a_{p_{1}, p_{2}}\right),
$$

the matrices of the order $p_{2}$, we have

$$
\begin{aligned}
\mathcal{S}_{4} & =\left[\begin{array}{ll}
\mathrm{e}^{\mathrm{i} x_{1}} G_{1} \\
\mathrm{e}^{-\mathrm{i} x_{1}} G_{1}^{*}
\end{array}\right. \\
& =\mathcal{S}_{4}^{\prime}+\mathcal{S}_{4}^{\prime \prime}+\mathcal{S}_{4}^{\prime \prime \prime},
\end{aligned}
$$


SO

$$
\mathcal{S}\left(x_{1}, x_{2}\right)=\mathcal{S}_{1}+\mathcal{S}_{3}+\mathcal{S}_{4}^{\prime}+\mathcal{S}_{4}^{\prime \prime}+\mathcal{S}_{4}^{\prime \prime \prime}+\sum_{j=1}^{p_{1}-1} E_{j} .
$$

As the next step toward (3.2), let us turn to the absolute value $|A|=\left(A^{*} A\right)^{1 / 2}$ of a matrix $A$, which occurs in the polar representation $A=V|A|$. For an Hermitian matrix $A$, its absolute value can be defined as follows. Denote by $P_{+}\left(P_{-}\right)$the projection onto the nonnegative (negative) eigenspace of $A$. Then

$$
A=A_{+}-A_{-}, \quad|A|=A_{+}+A_{-}, \quad A_{ \pm}:= \pm P_{ \pm} A \geq 0 .
$$

It is clear from this definition that

$$
-|A| \leq A \leq|A|, \quad-\sum_{j=1}^{N}\left|A_{j}\right| \leq \sum_{j=1}^{N} A_{j} \leq \sum_{j=1}^{N}\left|A_{j}\right| .
$$

We apply (3.6) to decomposition (3.5) to obtain (3.2) with

$$
\mathcal{S}_{ \pm}:=\mathcal{S}_{1} \pm D, \quad D:=\left|\mathcal{S}_{3}\right|+\left|\mathcal{S}_{4}^{\prime}\right|+\left|\mathcal{S}_{4}^{\prime \prime}\right|+\left|\mathcal{S}_{4}^{\prime \prime \prime}\right|+\sum_{j=1}^{p_{1}-1}\left|E_{j}\right|
$$

and so, by (3.3),

$$
|\sigma(J)|=|\sigma(M(\mathcal{S}))| \leq 2 \operatorname{tr} D .
$$

The value on the right side of (3.7) can be computed explicitly

$$
\operatorname{tr} D:=\operatorname{tr}\left|\mathcal{S}_{3}\right|+\operatorname{tr}\left|\mathcal{S}_{4}^{\prime}\right|+\operatorname{tr}\left|\mathcal{S}_{4}^{\prime \prime}\right|+\operatorname{tr}\left|\mathcal{S}_{4}^{\prime \prime \prime}\right|+\sum_{j=1}^{p_{1}-1} \operatorname{tr}\left|E_{j}\right| .
$$

Indeed,

$$
\begin{aligned}
& \left|\mathcal{S}_{3}\right|=\operatorname{diag}\left(\left|\mathcal{B}_{1}^{2}\right|, \ldots,\left|\mathcal{B}_{p_{1}}^{2}\right|\right), \quad\left|\mathcal{B}_{j}^{2}\right|=\operatorname{diag}\left(\left|b_{j, p_{2}}\right|, 0, \ldots, 0,\left|b_{j, p_{2}}\right|\right) ; \\
& \operatorname{tr}\left|\mathcal{S}_{3}\right|=\sum_{i=1}^{p_{1}} \operatorname{tr}\left|\mathcal{B}_{i}^{2}\right|=2 \sum_{i=1}^{p_{1}}\left|b_{i, p_{2}}\right| .
\end{aligned}
$$

Next, since

$$
\begin{aligned}
\left|G_{1}\right| & =\left(G_{1}^{*} G_{1}\right)^{1 / 2}=\operatorname{diag}\left(\left|\alpha_{p_{1}, 1}\right|,\left|\alpha_{p_{1}, 2}\right|, \ldots,\left|\alpha_{p_{1}, p_{2}}\right|\right), \\
\left|G_{1}^{*}\right| & =\left(G_{1} G_{1}^{*}\right)^{1 / 2}=\operatorname{diag}\left(\left|\alpha_{p_{1}, p_{2}}\right|,\left|\alpha_{p_{1}, 1}\right|, \ldots,\left|\alpha_{p_{1}, p_{2}-1}\right|\right),
\end{aligned}
$$

we see that

$$
\operatorname{tr}\left|\mathcal{S}_{4}^{\prime}\right|=2 \operatorname{tr}\left|G_{1}\right|=2 \sum_{i=1}^{p_{2}}\left|\alpha_{p_{1}, i}\right|, \quad \operatorname{tr}\left|\mathcal{S}_{4}^{\prime \prime}\right|=2 \operatorname{tr}\left|G_{1}^{*}\right|=2 \sum_{i=1}^{p_{2}}\left|\alpha_{p_{1}, i}\right| .
$$

Clearly,

$$
\operatorname{tr}\left|\mathcal{S}_{4}^{\prime \prime \prime}\right|=2 \operatorname{tr}\left|G_{2}\right|=2 \sum_{i=1}^{p_{2}}\left|a_{p_{1}, i}\right| .
$$


Finally,

$$
\begin{aligned}
& \left|E_{j}\right|=\operatorname{diag}\left(0, \ldots, 0,\left|\mathcal{A}_{j}^{2}\right|,\left|\mathcal{A}_{j}^{2}\right|, 0, \ldots, 0\right), \\
& \left|\mathcal{A}_{j}^{2}\right|=\operatorname{diag}\left(\left|\alpha_{j, p_{2}}\right|, 0, \ldots, 0,\left|\alpha_{j, p_{2}}\right|\right), \quad j=1,2, \ldots, p_{1}-1,
\end{aligned}
$$

and so

$$
\operatorname{tr}\left|E_{j}\right|=2 \operatorname{tr}\left|\mathcal{A}_{j}^{2}\right|=4\left|\alpha_{j, p_{2}}\right|, \quad \sum_{j=1}^{p_{1}-1} \operatorname{tr}\left|E_{j}\right|=4 \sum_{j=1}^{p_{1}-1}\left|\alpha_{j, p_{2}}\right| .
$$

A combination of the above equalities gives

$$
\operatorname{tr}|D|=2 \sum_{j=1}^{p_{1}}\left(\left|b_{j, p_{2}}\right|+2\left|\alpha_{j, p_{2}}\right|\right)+2 \sum_{i=1}^{p_{2}}\left(\left|a_{p_{1}, i}\right|+2\left|\alpha_{p_{1}, i}\right|\right)-4\left|\alpha_{p_{1}, p_{2}}\right|,
$$

and hence

$$
|\sigma(J)| \leq 2 \operatorname{tr} D=4 \sum_{j=1}^{p_{1}}\left(\left|b_{j, p_{2}}\right|+2\left|\alpha_{j, p_{2}}\right|\right)+4 \sum_{i=1}^{p_{2}}\left(\left|a_{p_{1}, i}\right|+2\left|\alpha_{p_{1}, i}\right|\right)-8\left|\alpha_{p_{1}, p_{2}}\right| .
$$

Let us note that there is nothing special in the choice of indices $p_{1}, p_{2}$ in the latter inequality. Indeed, it is not hard to find a unitary operator (block-shift) $W=W_{m, n}$ on $\ell^{2}\left(\mathbb{Z}, \ell^{2}(\mathbb{Z})\right.$ ) so that $\hat{J}:=W J W^{*}$ is again the block-Jacobi operator (1.2) with the shifted entries. Since $\sigma(J)=\sigma(\hat{J})$, the result follows. The proof of Theorem 1.1 is complete.

Corollary 1.2 is a special case with $\alpha_{i, k}=0$ for $L$, and $a_{i, k}=b_{i, k}=1$ for $H$.

Remark 3.1. In the case when either of $p_{1}, p_{2}$ equals 2 , the argument is the same with expressions $(2.7),(2.8)$ in place of $(2.1)-(2.3)$. In particular, for $p_{1}=p_{2}=2$ we have

$$
\frac{1}{4}|\sigma(J)| \leq\left|a_{2,1}\right|+\left|a_{2,2}\right|+\left|b_{1,2}\right|+\left|b_{2,2}\right|+2\left(\left|\alpha_{1,2}\right|+\left|\alpha_{2,1}\right|+\left|\alpha_{2,2}\right|\right) .
$$

Remark 3.2. In [8, Theorem 3.11] H. Krüger obtained a uniform upper bound for the length of the spectral bands $\Lambda_{k}$ (2.9) for multidimensional periodic Schrödinger operators, which for $d=2$ reads

$$
\left|\Lambda_{k}\right| \leq 4 \pi\left(\frac{1}{p_{1}}+\frac{1}{p_{2}}\right)
$$

The latter implies the bound for the length of the whole spectrum

$$
|\sigma(H)| \leq 4 \pi\left(p_{1}+p_{2}\right) .
$$

Compared to Corollary 1.2, an extra factor $\pi$ is on the right side of (3.8).

Remark 3.3. There is yet another way to obtain the upper bound for the length of $\sigma(J)$, based on Gershgorin's theorem [6, Theorem 6.1.1]. Indeed, let the symbol

$$
\mathcal{S}\left(x_{1}, x_{2}\right)=\left\|s_{i, j}\left(x_{1}, x_{2}\right)\right\|_{i, j=1}^{p} .
$$

Denote by $\mathcal{G}_{n}$ the Gershgorin disk (interval)

$$
\mathcal{G}_{n}\left(x_{1}, x_{2}\right):=\left\{\lambda \in \mathbb{R}:\left|\lambda-s_{n n}\left(x_{1}, x_{2}\right)\right| \leq \sum_{j \neq n}\left|s_{n, j}\left(x_{1}, x_{2}\right)\right|, n=1, \ldots, p\right\} .
$$


It is important that in our case $s_{n, n}$ and $\left|s_{n, j}\right|$ are constants, that is, do not depend on $x_{1}, x_{2}$, see (2.1)-(2.3), and so do the Gershgorin intervals $\mathcal{G}_{n}$. By Gershgorin's theorem, the spectral bands $(2.9)$

$$
\Lambda_{k} \subset \mathcal{G}:=\bigcup_{n=1}^{p} \mathcal{G}_{n}
$$

and so

$$
|\sigma(J)| \leq|\mathcal{G}| \leq \sum_{n=1}^{p}\left|\mathcal{G}_{n}\right| \leq 2 \sum_{i \neq j}\left|s_{i, j}\right|,
$$

the two times sum of all off-diagonal entries of the symbol $\mathcal{S}$. In view of $(2.3)$, the latter leads to the bound

$$
|\sigma(J)| \leq \tilde{R}:=2 \sum_{i=1}^{p_{1}} \sum_{j=1}^{p_{2}}\left(4\left|\alpha_{i, j}\right|+2\left|a_{i, j}\right|+2\left|b_{i, j}\right|\right) .
$$

Compared to (1.7), there is a double sum on the right side (3.9). It is easy to see that $\tilde{R} \geq \max _{(m, n) \in \mathbb{T}^{2}} R_{m, n}$, so the bound (1.7)-(1.8) is better than (3.9).

We proceed with two examples which illustrate the optimal character of the bound in Theorem 1.1 .

Example 3.4. Assume that

$$
A_{q} \equiv 0, \quad B_{q}=S+S^{-1}+4 q I, \quad q=1, \ldots, p_{1}, \quad B_{q+p_{1}}=B_{q},
$$

$S$ is the standard shift in $\ell^{2}(\mathbb{Z})$. In other words, we have

$$
a_{q, r}=\alpha_{q, r} \equiv 0, \quad b_{q, r} \equiv 1, \quad c_{q, r}=4 q, \quad q=1, \ldots, p_{1}, \quad c_{q+p_{1}, r}=c_{q, r} .
$$

Now the block-Jacobi operator $J(1.1)$ is block-diagonal, $J=\operatorname{diag}\left(B_{q}\right)_{q \in \mathbb{Z}}$. Since $\sigma\left(B_{q}\right)=$ $[4 q-2,4 q+2]$, we see that $\sigma\left(B_{j}\right) \cap \sigma\left(B_{k}\right)$ is at most one point set for $j, k=1, \ldots, p_{1}, j \neq k$. Hence,

$$
\sigma(J)=\bigcup_{q=1}^{p_{1}} \sigma\left(B_{q}\right)=\left[2,2+4 p_{1}\right] \quad \Rightarrow \quad|\sigma(J)|=4 p_{1},
$$

and the factor 4 in Theorem 1.1 is optimal.

Example 3.5. Assume that $A_{q} \equiv I, B_{q} \equiv B$, where $B=\operatorname{diag}\left(\beta_{r}\right)_{r \in \mathbb{Z}}$ is a diagonal periodic matrix with

$$
\beta_{r}=4 r, \quad r=1,2, \ldots, p_{2}, \quad \beta_{r+p_{2}}=\beta_{r} .
$$

In this case $J=J(\{B\},\{I\})$ is unitarily equivalent to the orthogonal sum

$$
J \simeq \bigoplus_{k=1}^{p_{2}}\left(J^{0}+4 k I\right)
$$

$J^{0}$ is the discrete Laplacian in $\ell^{2}(\mathbb{Z})$, so again

$$
\sigma(J)=\bigcup_{k=1}^{p_{2}}[4 k-2,4 k+2]=\left[2,2+4 p_{2}\right] \quad \Rightarrow \quad|\sigma(J)|=4 p_{2} .
$$

This example again illustrates the optimal character of Theorem 1.1. 


\section{Examples of explicitly computed spectra}

The discrete version of the famous Bethe-Sommerfeld conjecture concerns the structure of the spectrum of periodic, discrete Schrödinger operators $H$ (1.6) (and their multidimensional analogues). It claims that for small enough potentials $\left\{c_{i k}\right\}$ such spectrum is a union of at most two closed intervals, with the gap open at the zero energy. Moreover, the spectrum is a single interval as long as at least one number $p_{1}, p_{2}$ is odd. The result was proved for $d=2$ in [2], with a partial case for coprime periods in [8], and for an arbitrary dimension $d \geq 2$ in [5]. This result contrasts strongly with the one-dimensional case, wherein a generic $p$-periodic operator has the spectrum with $p-1$ gaps open.

It turns out that Corollary 2.3 enables one to find the spectra for certain (2,2)-periodic discrete Schrödinger operators $H$ (1.6), with not necessarily small potentials. Indeed, by Remark 2.2 , the symbol is now

$$
\mathcal{S}\left(x_{1}, x_{2}\right)=\left[\begin{array}{cc}
\mathcal{B}_{1} & \tau\left(x_{1}\right) I_{2} \\
\tau\left(-x_{1}\right) I_{2} & \mathcal{B}_{2}
\end{array}\right]
$$

with $\tau(x):=1+\mathrm{e}^{\mathrm{i} x}$,

$$
\mathcal{B}_{1}=\left[\begin{array}{cc}
c_{11} & \tau\left(x_{2}\right) \\
\tau\left(-x_{2}\right) & c_{12}
\end{array}\right], \quad \mathcal{B}_{2}=\left[\begin{array}{cc}
c_{21} & \tau\left(x_{2}\right) \\
\tau\left(-x_{2}\right) & c_{22}
\end{array}\right]
$$

The characteristic polynomial of the symbol is

$$
\mathcal{D}(\lambda)=\operatorname{det}\left(\mathcal{S}\left(x_{1}, x_{2}\right)-\lambda I_{4}\right)=\left|\begin{array}{cc}
\mathcal{B}_{1}-\lambda I_{2} & \tau\left(x_{1}\right) I_{2} \\
\tau\left(-x_{1}\right) I_{2} & \mathcal{B}_{2}-\lambda I_{2}
\end{array}\right|
$$

To compute this determinant we apply the Schur formula, which reduces determinants of order $2 n$ to ones of order $n$ (see, e.g., [4, Section II.5]). Precisely, if $A_{1}, A_{2}, A_{3}, A_{4}$ are $n \times n$ matrices, and $A_{1} A_{3}=A_{3} A_{1}$, then

$$
\left|\begin{array}{ll}
A_{1} & A_{2} \\
A_{3} & A_{4}
\end{array}\right|=\operatorname{det}\left(A_{1} A_{4}-A_{3} A_{2}\right)
$$

Hence,

$$
\mathcal{D}(\lambda)=\operatorname{det} D(\lambda), \quad D(\lambda)=\left(\left(\mathcal{B}_{1}-\lambda I_{2}\right)\left(\mathcal{B}_{2}-\lambda I_{2}\right)-\left|\tau\left(x_{1}\right)\right|^{2} I_{2}\right) .
$$

Example 4.1. Let

$$
c_{i j}=(-1)^{i+j} c, \quad i, j=1,2, \quad c>0 .
$$

We find explicitly the spectrum of the corresponding operator $H$ (1.6):

$$
\sigma(H)=\left[-\sqrt{c^{2}+16},-c\right] \cup\left[c, \sqrt{c^{2}+16}\right] .
$$

Indeed,

$$
\begin{aligned}
\left(\mathcal{B}_{1}-\lambda I_{2}\right)\left(\mathcal{B}_{2}-\lambda I_{2}\right) & =\left[\begin{array}{cc}
c-\lambda & \tau\left(x_{2}\right) \\
\tau\left(-x_{2}\right) & -c-\lambda
\end{array}\right]\left[\begin{array}{cc}
-c-\lambda & \tau\left(x_{2}\right) \\
\tau\left(-x_{2}\right) & c-\lambda
\end{array}\right] \\
& =\left[\begin{array}{cc}
\lambda^{2}-c^{2}+\left|\tau\left(x_{2}\right)\right|^{2} & 2 \tau\left(x_{2}\right)(c-\lambda) \\
-2 \tau\left(-x_{2}\right)(c+\lambda) & \lambda^{2}-c^{2}+\left|\tau\left(x_{2}\right)\right|^{2}
\end{array}\right],
\end{aligned}
$$


and, by (4.1),

$$
\begin{aligned}
\mathcal{D}(\lambda) & =\left(\lambda^{2}-c^{2}+\left|\tau\left(x_{2}\right)\right|^{2}-\left|\tau\left(x_{1}\right)\right|^{2}\right)^{2}-4\left|\tau\left(x_{2}\right)\right|^{2}\left(\lambda^{2}-c^{2}\right) \\
& =\left(\zeta+\left|\tau\left(x_{2}\right)\right|^{2}-\left|\tau\left(x_{1}\right)\right|^{2}\right)^{2}-4\left|\tau\left(x_{2}\right)\right|^{2} \zeta \\
& =\left(\zeta-\left|\tau\left(x_{2}\right)\right|^{2}-\left|\tau\left(x_{1}\right)\right|^{2}\right)^{2}-4\left|\tau\left(x_{2}\right)\right|^{2}\left|\tau\left(x_{1}\right)\right|^{2}, \quad \zeta=\lambda^{2}-c^{2} .
\end{aligned}
$$

So, the eigenvalues of the symbol are

$$
\begin{aligned}
& \lambda_{1}\left(x_{1}, x_{2}\right)=\sqrt{c^{2}+\left(\left|\tau\left(x_{2}\right)\right|+\left|\tau\left(x_{1}\right)\right|\right)^{2}}, \\
& \lambda_{2}\left(x_{1}, x_{2}\right)=\sqrt{c^{2}+\left(\left|\tau\left(x_{2}\right)\right|-\left|\tau\left(x_{1}\right)\right|\right)^{2}}, \\
& \lambda_{3}\left(x_{1}, x_{2}\right)=-\lambda_{2}\left(x_{1}, x_{2}\right), \quad \lambda_{4}\left(x_{1}, x_{2}\right)=-\lambda_{1}\left(x_{1}, x_{2}\right) .
\end{aligned}
$$

The spectral bands (3.1) are

$$
\Lambda_{1}=\left[c, \sqrt{c^{2}+16}\right], \quad \Lambda_{2}=\left[c, \sqrt{c^{2}+4}\right], \quad \Lambda_{3}=-\Lambda_{2}, \quad \Lambda_{4}=-\Lambda_{1},
$$

as claimed.

Remark 4.2. The latter example is a key one in [8], wherein it is shown that there is a gap in the spectrum for all $c>0$. For the similar computation of the spectrum for various lattices see [3, Theorem 3.5].

Example 4.3. Consider now a two-parameter family of $(2,2)$-periodic Schrödinger operators with

$$
c_{11}=-c_{22}=: c_{1}, \quad c_{12}=-c_{21}=: c_{2}, \quad c_{1}, c_{2}>0 .
$$

Denote $\beta\left(x_{1}, x_{2}\right):=\left|\tau\left(x_{2}\right)\right|^{2}-\left|\tau\left(x_{1}\right)\right|^{2}$, so for $D$ in (4.1) one has

$$
D(\lambda)=\left[\begin{array}{cc}
\left(\lambda-c_{1}\right)\left(\lambda+c_{2}\right)+\beta\left(x_{1}, x_{2}\right) & -2 \lambda \tau\left(x_{2}\right) \\
-2 \lambda \tau\left(-x_{2}\right) & \left(\lambda-c_{2}\right)\left(\lambda+c_{1}\right)+\beta\left(x_{1}, x_{2}\right)
\end{array}\right]
$$

and hence

$$
\mathcal{D}(\lambda)=\left(\lambda^{2}-c_{1}^{2}\right)\left(\lambda^{2}-c_{2}^{2}\right)+2 \beta\left(x_{1}, x_{2}\right)\left(\lambda^{2}-c_{1} c_{2}\right)+\beta^{2}\left(x_{1}, x_{2}\right)-4 \lambda^{2}\left|\tau\left(x_{2}\right)\right|^{2} .
$$

The roots of this biquadratic polynomial can be found explicitly. For $z=\lambda^{2}, \mathcal{D}_{1}(z)=\mathcal{D}(\lambda)$, we have

$$
\begin{aligned}
\mathcal{D}_{1}(z) & =z^{2}-z\left(c_{1}^{2}+c_{2}^{2}+4\left|\tau\left(x_{2}\right)\right|^{2}-2 \beta\left(x_{1}, x_{2}\right)\right)+\left(\beta\left(x_{1}, x_{2}\right)-c_{1} c_{2}\right)^{2} \\
& =z^{2}-z\left(c_{1}^{2}+c_{2}^{2}+2\left|\tau\left(x_{2}\right)\right|^{2}+2\left|\tau\left(x_{1}\right)\right|^{2}\right)+\left(\beta\left(x_{1}, x_{2}\right)-c_{1} c_{2}\right)^{2} \\
& =z^{2}-2 A z+B
\end{aligned}
$$

where

$$
A:=\frac{c_{1}^{2}+c_{2}^{2}}{2}+\left|\tau\left(x_{1}\right)\right|^{2}+\left|\tau\left(x_{2}\right)\right|^{2}, \quad B:=\left(\beta\left(x_{1}, x_{2}\right)-c_{1} c_{2}\right)^{2} \geq 0 .
$$

The roots $z_{ \pm}$of $\mathcal{D}_{1}$ are

$$
\begin{aligned}
& z_{ \pm}\left(x_{1}, x_{2}\right)=A \pm \sqrt{D} \\
& D=A^{2}-B=\left[\frac{\left(c_{1}-c_{2}\right)^{2}}{2}+2\left|\tau\left(x_{2}\right)\right|^{2}\right]\left[\frac{\left(c_{1}+c_{2}\right)^{2}}{2}+2\left|\tau\left(x_{1}\right)\right|^{2}\right] \geq 0
\end{aligned}
$$


Hence, $0 \leq z_{-}\left(x_{1}, x_{2}\right) \leq z_{+}\left(x_{1}, x_{2}\right)$ for each $\left(x_{1}, x_{2}\right) \in \mathbb{T}^{2}$, and

$$
\lambda_{1}\left(x_{1}, x_{2}\right)=-\lambda_{4}\left(x_{1}, x_{2}\right)=\sqrt{z_{+}\left(x_{1}, x_{2}\right)}, \quad \lambda_{2}\left(x_{1}, x_{2}\right)=-\lambda_{3}\left(x_{1}, x_{2}\right)=\sqrt{z_{-}\left(x_{1}, x_{2}\right)} .
$$

We begin with the interior gap at the origin. Clearly, this gap is open if and only if

$$
l_{2}=\min _{\mathbb{T}^{2}} z_{-}\left(x_{1}, x_{2}\right)>0 .
$$

The latter is equivalent to

$$
\min _{\mathbb{T}^{2}} B\left(x_{1}, x_{2}\right)=\min _{\mathbb{T}^{2}}\left(2\left(\cos x_{1}-\cos x_{2}\right)-c_{1} c_{2}\right)^{2}>0 .
$$

So, the interior gap at the origin is open if and only if $c_{1} c_{2}>4$.

There are another two gaps (possibly closed), symmetric with respect to the origin, which we refer to as "exterior gaps". Such gaps are open if and only if

$$
\max _{\mathbb{T}^{2}} z_{-}\left(x_{1}, x_{2}\right)<\min _{\mathbb{T}^{2}} z_{+}\left(x_{1}, x_{2}\right) .
$$

It is easy to see that

$$
\min _{\mathbb{T}^{2}} z_{+}\left(x_{1}, x_{2}\right)=z_{+}(\pi, \pi)=\frac{c_{1}^{2}+c_{2}^{2}}{2}+\frac{\left|c_{1}-c_{2}\right|}{2}=\max \left(c_{1}^{2}, c_{2}^{2}\right) .
$$

On the other hand, it is a matter of elementary (though lengthy) calculus to check that

$$
\max _{\mathbb{T}^{2}} z_{-}\left(x_{1}, x_{2}\right)=z_{-}(0, \pi)=\frac{c_{1}^{2}+c_{2}^{2}}{2}+4-\frac{\left|c_{1}-c_{2}\right|}{2} \sqrt{\left(c_{1}+c_{2}\right)^{2}+16}
$$

So, (4.2) is equivalent to

$$
\frac{c_{1}^{2}+c_{2}^{2}}{2}+4-\frac{\left|c_{1}-c_{2}\right|}{2} \sqrt{\left(c_{1}+c_{2}\right)^{2}+16}<\max \left(c_{1}^{2}, c_{2}^{2}\right) .
$$

A simple sufficient condition for the exterior gaps to be open is

$$
c_{1}^{2}>c_{2}^{2}+8 \text {. }
$$

To summarize, the conditions (4.3) and $c_{1} c_{2}>4$ ensure the existence of all three gaps, under conditions (4.3) and $c_{1} c_{2} \leq 4$ the exterior gaps are open. When $c_{1}=c_{2}+\varepsilon, \varepsilon>0$ small enough, and $c_{2}>2$ only interior gap is open.

We complete with a discrete Schrödinger operator (1.6), which has maximal number of gaps in its spectrum.

Example 4.4. Let $H$ be a $\left(p_{1}, p_{2}\right)$-periodic, discrete Schrödinger operator with the potential

$$
c_{i, j}=\frac{(i-1) p_{2}+j}{\varepsilon}, \quad i=1, \ldots, p_{1}, \quad j=1, \ldots, p_{2}, \quad 0<\varepsilon<\frac{1}{8} .
$$

The symbol is now

$$
\begin{aligned}
& \mathcal{S}\left(x_{1}, x_{2}\right)= {\left[\begin{array}{ccccc}
\mathcal{B}_{1} & I_{p_{2}} & & & \mathrm{e}^{\mathrm{i} x_{1}} I_{p_{2}} \\
I_{p_{2}} & \mathcal{B}_{2} & I_{p_{2}} & & \\
& \ddots & \ddots & \ddots & \\
& & I_{p_{2}} & \mathcal{B}_{p_{1}-1} & I_{p_{2}} \\
\mathrm{e}^{-\mathrm{i} x_{1}} I_{p_{2}} & & I_{p_{2}} & \mathcal{B}_{p_{1}}
\end{array}\right], } \\
& \mathcal{B}_{n}\left(x_{2}\right)=\left[\begin{array}{ccccc}
\frac{(n-1) p_{2}+1}{\varepsilon} & 1 & & & \mathrm{e}^{\mathrm{i} x_{2}} \\
1 & \frac{(n-1) p_{2}+2}{\varepsilon} & 1 & & \\
& \ddots & \ddots & \ddots & \\
& & 1 & \frac{n p_{2}-1}{\varepsilon} & 1 \\
\mathrm{e}^{-\mathrm{i} x_{2}} & & & 1 & \frac{n p_{2}}{\varepsilon}
\end{array}\right], \quad n=1,2, \ldots, p_{1} .
\end{aligned}
$$


The Gershgorin intervals (see Remark 3.3) take the form

$$
\mathcal{G}_{k}=\left[\frac{k}{\varepsilon}-l, \frac{k}{\varepsilon}+l\right], \quad k=1,2, \ldots, p, \quad l=3 \text { or } 4
$$

In any event, such intervals are disjoint for $\varepsilon<1 / 8$, and, by Gershgorin's theorem, each $\mathcal{G}_{k}$ contains exactly one eigenvalue $\lambda_{k}\left(x_{1}, x_{2}\right)$ of the symbol for all $\left(x_{1}, x_{2}\right) \in \mathbb{T}^{2}$. So, the spectral bands $\Lambda_{k} \subset \mathcal{G}_{k}$ and, by Corollary 2.3, there are $p-1$ gaps in the spectrum of $H$, as claimed.

\section{Acknowledgements}

We thank the anonymous referees for their valuable remarks which improved the paper significantly.

\section{References}

[1] Berezans'kiǔ Ju.M., Expansions in eigenfunctions of selfadjoint operators, Translations of Mathematical Monographs, Vol. 17, Amer. Math. Soc., Providence, R.I., 1968.

[2] Embree M., Fillman J., Spectra of discrete two-dimensional periodic Schrödinger operators with small potentials, arXiv:1701.00863.

[3] Fillman J., Han R., Discrete Bethe-Sommerfeld conjecture for triangular, square, and hexagonal lattices, arXiv:1806.01988.

[4] Gantmacher F.R., The theory of matrices, AMS Chelsea Publishing, Providence, RI, 1998.

[5] Han R., Jitomirskaya S., Discrete Bethe-Sommerfeld conjecture, Comm. Math. Phys. 361 (2018), 205-216, arXiv:1707.03482.

[6] Horn R.A., Johnson C.R., Matrix analysis, Cambridge University Press, Cambridge, 1985.

[7] Korotyaev E., Krasovsky I.V., Spectral estimates for periodic Jacobi matrices, Comm. Math. Phys. 234 (2003), 517-532, arXiv:math.SP/0205319.

[8] Krüger H., Periodic and limit-periodic discrete Schrödinger operators, arXiv:1108.1584.

[9] Kutsenko A.A., Estimates of parameters for conformal mappings related to a periodic Jacobi matrix, J. Math. Sci. 134 (2006), 2295-2304.

[10] Kutsenko A.A., On the measure of the spectrum of direct integrals, Banach J. Math. Anal. 9 (2015), 1-8, arXiv:1212.0235.

[11] Kutsenko A.A., Sharp spectral estimates for periodic matrix-valued Jacobi operators, in Mathematical Technology of Networks, Springer Proc. Math. Stat., Vol. 128, Springer, Cham, 2015, 133-136, arXiv:1007.5412.

[12] Simon B., Szegö's theorem and its descendants. Spectral theory for $L^{2}$ perturbations of orthogonal polynomials, M.B. Porter Lectures, Princeton University Press, Princeton, NJ, 2011. 